\title{
Fat misbehaving in the abdominal cavity: a pictorial essay
}

\author{
Hannes Devos ${ }^{\mathrm{B}, \mathrm{D}, \mathrm{E}}$, Lode Goethals ${ }^{\mathrm{E}}$, Dries Belsack ${ }^{\mathrm{B}}$, Yannick De Brucker ${ }^{\mathrm{E}}$, Gert-Jan Allemeersch ${ }^{\mathrm{F}}$, Bart Ilsen ${ }^{\mathrm{D}}$, \\ Frederik Vandenbroucke ${ }^{A}$, Johan de Mey ${ }^{A}$
}

UZ Brussel, Belgium

\begin{abstract}
Intra-abdominal fat is abundantly present in both the peritoneum and retroperitoneum. Fat necrosis or inflammation are common findings in abdominal imaging. The most common pathologies that we encounter are epiploic appendagitis, omental infarction, mesenteric panniculitis, and encapsulated fat necrosis. Less common entities that can occur are pancreatic saponification, heterotopic mesenteric ossification, and pseudolipoma of the capsule of Glisson. These entities can mimic more urgent pathologies such as appendicitis, diverticulitis, or malignancies.
\end{abstract}

Key words: necrosis, inflammation, computed tomography, abdominal imaging.

\section{Introduction}

Intra-abdominal fat is a metabolically active tissue that is abundantly present in both the retroperitoneal and intraperitoneal compartments. Lesions originating from the abdominal fat are a common finding in abdominal imaging. They may cause abdominal pain and have a clinical presentation that resembles that of acute abdomen, or they may be asymptomatic. The greater omentum and the mesentery are the structures in the abdomen that contain the greatest amount of fat. It is important to localise lesions accurately to establish a differential diagnosis.

\section{Epiploic appendagitis}

Epiploic appendages are benign outpouchings of peritoneal fat and small blood vessels on the anti-mesenteric side of the colon. They arise from the serosal surface adjacent to the taeniae coli in the colon. They are most abundant in the left colon and cecum [1]. The rectum, on the other hand, does not contain any epiploic appendages. The typical imaging features include an oval lesion of several centimetres in length, with a peripheral rim of hyperattenuation and surrounding inflammatory changes, which abuts the anterior colon wall (Figures 1-3). A cen- tral dot of high attenuation may also be visualised, which represents a thrombosed vein or internal haemorrhage. Associated inflammation of the colon wall is usually not present $[2,3]$.

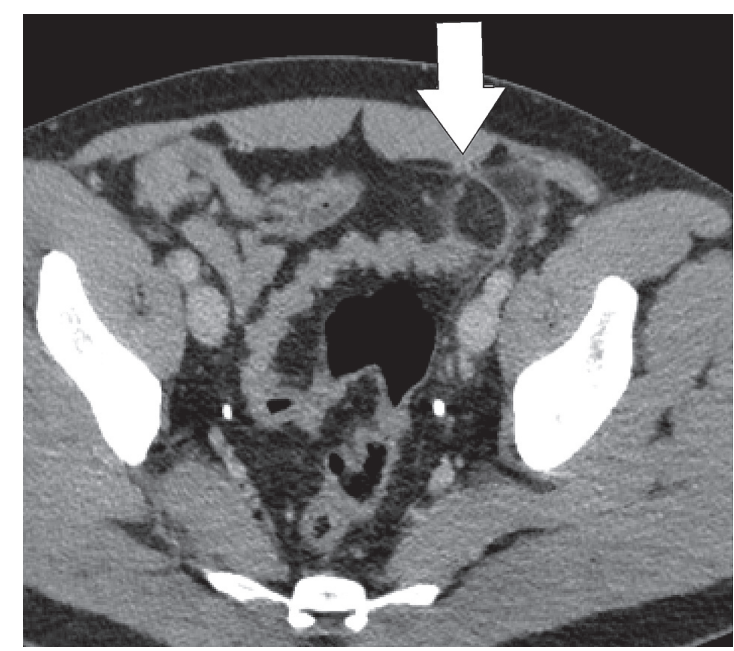

Figure 1. Epiploic appendagitis. Axial computed tomography image of a patient with left-sided flank pain. Presence of a fat-density, ovoid structure (white arrow) with a thin high-density rim, adjacent to the colon, characteristic for an epiploic appendagitis

Correspondence address:

Dr. Hannes Devos, UZ Brussel, Laarbeeklaan 101, 1090 Jette, Belgium, e-mail: hdevos@uzbrussel.be

Authors' contribution:

A Study design · B Data collection - C Statistical analysis · D Data interpretation - E Manuscript preparation · F Literature search · G Funds collection 


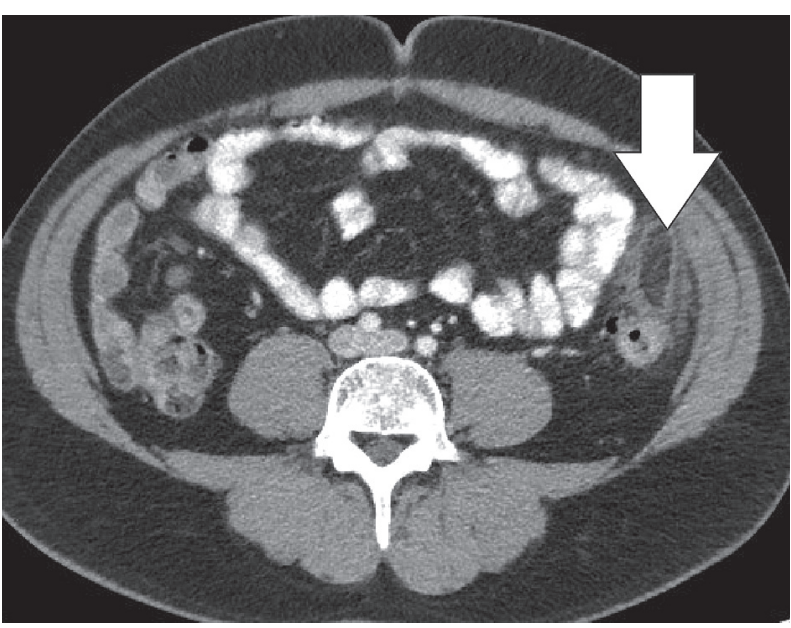

Figure 2. Epiploic appendagitis. Axial computed tomography image. Sometimes the lesion (white arrow) can become quite large. In this case the ring enhancing lesion was up to $4 \mathrm{~cm}$ in length

\section{Omental infarction}

The greater omentum is the largest peritoneal fold and consists of a double sheet of peritoneum. It continuous with the visceral peritoneal layers of the stomach and transverse colon. The greater omentum contains fat and blood vessels and is known as the 'policeman of the abdomen', with the purpose of containing the spread of peritoneal infections [4]. Omental infarction is typically triangular in shape and is most commonly encountered inferiorly of the liver. It is characteristically situated between the anterior abdominal wall and the transverse or ascending colon. Right-sided omentum has a greater incidence of infarction due to higher mobility and greater length. Both computed tomography (CT) and ultrasound show a heterogeneous soft-tissue mass (Figures 4, 5). In its early stages, omental infarction may manifest as subtle inflammatory changes in the fat anterior to the colon [5]. In most patients, omental infarction has a benign course that can be treated with analgesics and does not require

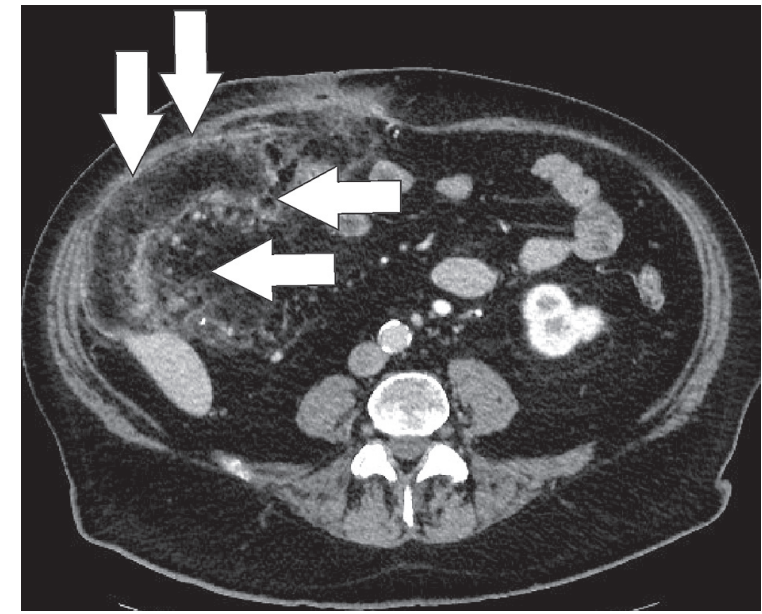

Figure 5. Omental infarction. Axial computed tomography image. A large, partially encapsulated infarction (white arrows) of the omentum on the right side secondary to an ileocaecal resection

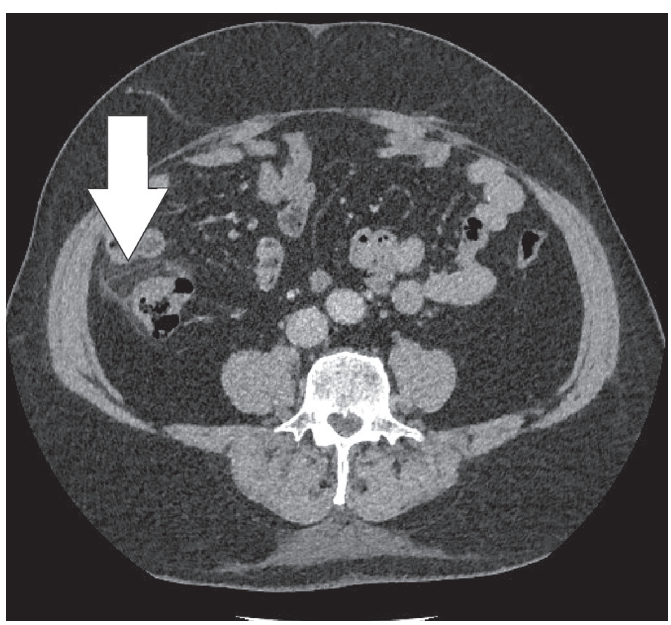

Figure 3. Epiploic appendagitis. Axial computed tomography image. The typical findings of appendagitis are sometimes absent, with merely a discrete fatty infiltration (white arrow) around the ascending colon

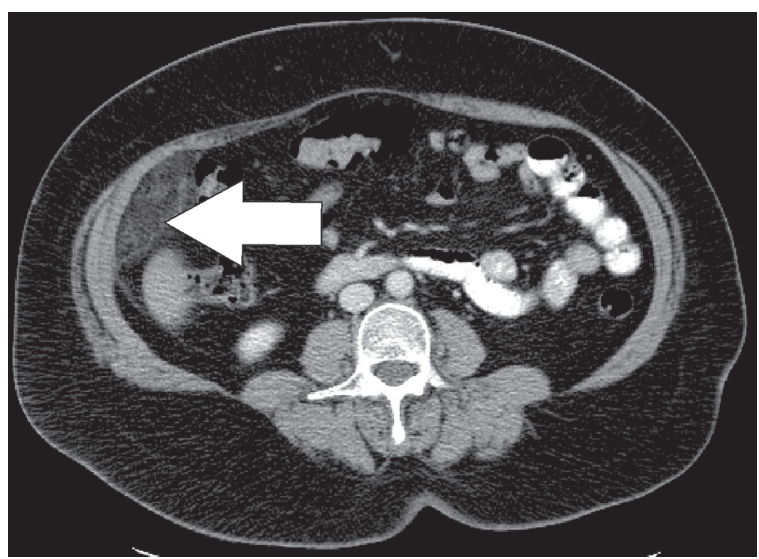

Figure 4. Omental infarction. Axial computed tomography image displays a diffuse infiltration of the omental fat (white arrow) inferior to the right liver lobe

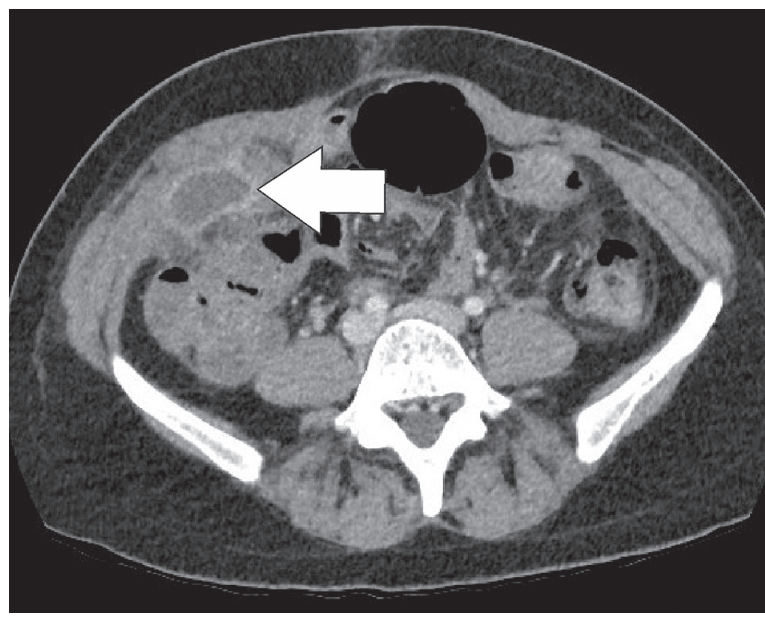

Figure 6. Omental infarction. Axial computed tomography (CT) image of a young woman that came to the ER with persistent right flank pain postpartum. Images show an encapsulated collection on the right side (white arrow), which was subsequently drained under (T-guidance. This case showed no bacterial growth from the cultures, revealing this abscess to be secondary to an omental infarction 
intervention [6]. The inflammatory response resolves with retraction and fibrosis leading to either complete healing or autoamputation [7]. Rare complications include bowel obstruction caused by adhesions and abscess formation (Figure 6) [8].

\section{Saponification secondary to pancreatitis}

Damaged pancreatic tissue can release lipolytic enzymes, which autodigest the pancreatic parenchyma and peripancreatic fat tissues. This in turn results in the activation of mediating factors of inflammation, mainly macrophages, which exaggerate the inflammatory response. As phospholipases and proteases attack the plasma membranes of fat cells, triglycerides are released and hydrolysed. The lysis of the fat cells causes the release of fatty acids that precipitate

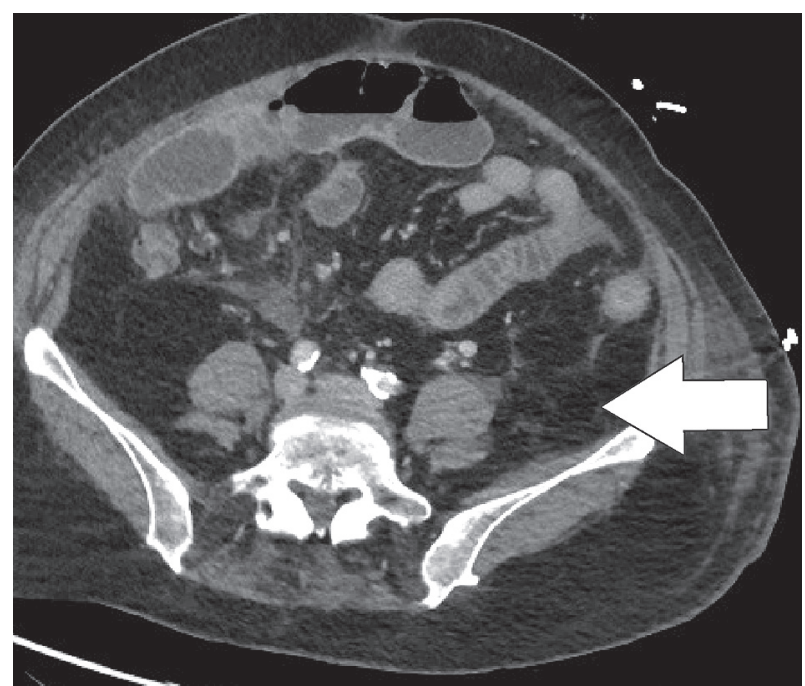

Figure 7. Fat saponification. Axial computed tomography image of a patient with acute necrotic pancreatitis. There is a subtle infiltration of the fat in the retroperitoneal space (white arrow)

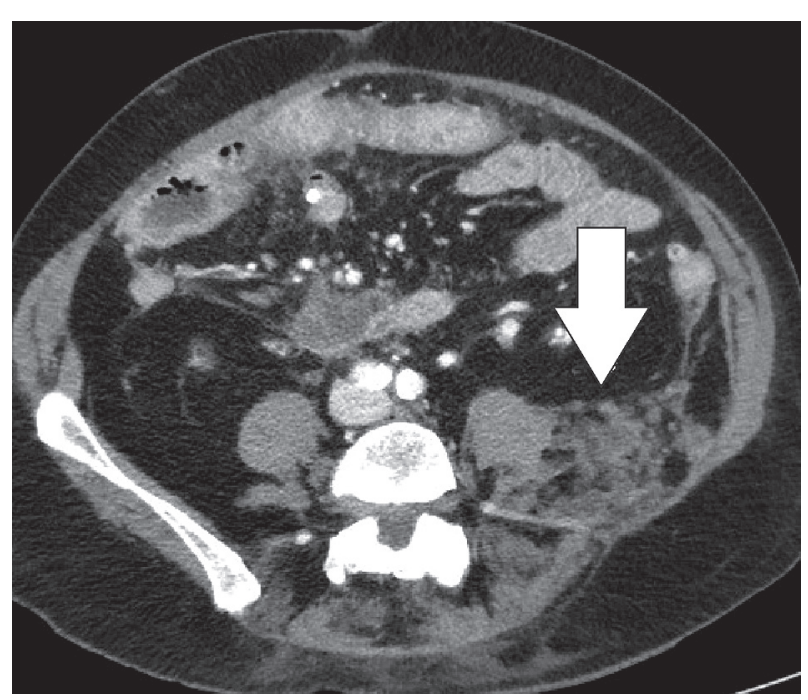

Figure 8. Fat saponification. Subsequent axial computed tomography images of the same patient, acquired 3 weeks later, demonstrate the onset of multiple soft-tissue nodules in the retroperitoneal space (white arrow)

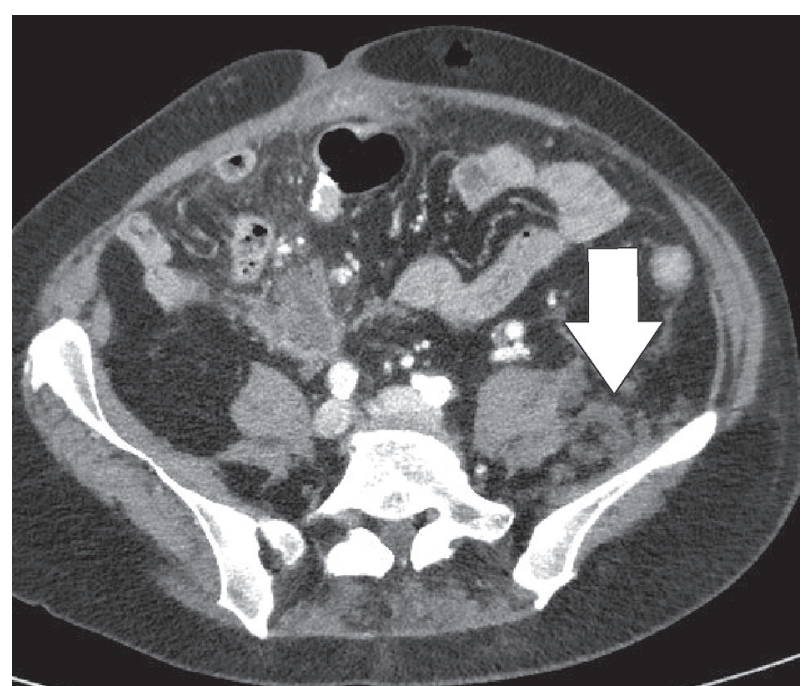

Figure 9. Fat saponification. Subsequent axial computed tomography images of the same patient, acquired 3 weeks later, demonstrate the onset of multiple soft-tissue nodules in the retroperitoneal space (white arrow)

with the serum calcium in the form of soap. This is the phenomenon known as fatty saponification $[9,10]$. After resolution of the acute phase of pancreatitis, scattered nodular lesions can be visualised in the retroperitoneum and mesentery (Figures 7-9) [6]. The clinical history or previous imaging studies may be necessary to differentiate pancreatic fat saponification from peritoneal carcinomatosis.

\section{Encapsulated fat necrosis}

Encapsulated fat necrosis is a benign condition that was first described in the breast in 1975, but it can occur anywhere in the body [11]. A variety of different names have already been used to describe these lesions. These terms include nodular-cystic fat necrosis, mobile encapsulated lipoma, encapsulated necrosis, or posttraumatic fat necrosis [12]. Encapsulated fat necrosis is thought to result from rapid infarction of adipose tissue secondary to trauma or interrupted vascularisation. This process initiates the organisation of the fatty tissue within a fibrous capsule [6,13]. Imaging features may vary, and mass effect can also be present (Figures 10-12). Sometimes the capsule will show enhancement after IV contrast administration. Therefore, the most important differential diagnosis is liposarcoma. However, fat necrosis does not show signs of organ invasion. Tenderness can also be present during physical examination. Temporal evolution may aid in the differentiation: encapsulated fat necrosis will decrease in size over time, whereas liposarcomas typically become larger (Figure 13) [6].

\section{Mesenteric panniculitis}

Mesenteric panniculitis is a benign and chronic inflammatory disease of unknown origin, which involves the fatty tissue of the mesentery of the small intestine and colon [14]. It can be categorised according to three pathological chang- 


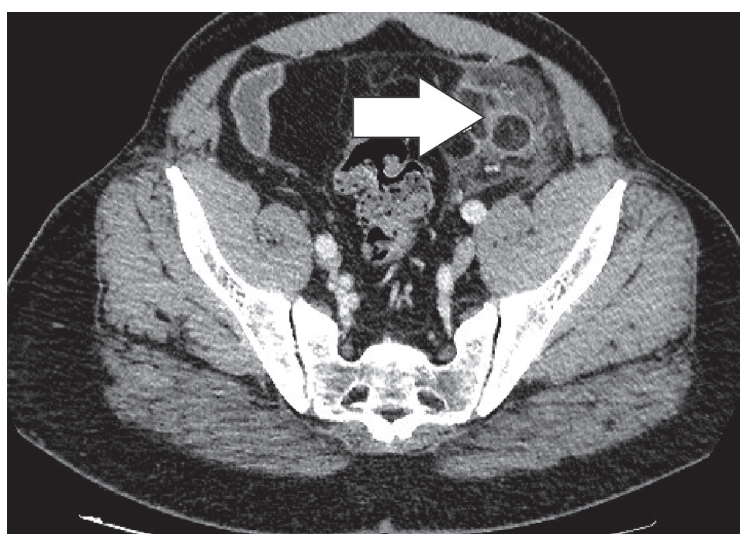

Figure 10. Encapsulated fat necrosis. Axial computed tomography image of a patient with left lower quadrant pain revealed the presence of an encapsulated lesion containing fat and surrounded by soft-tissue stranding (white arrow)

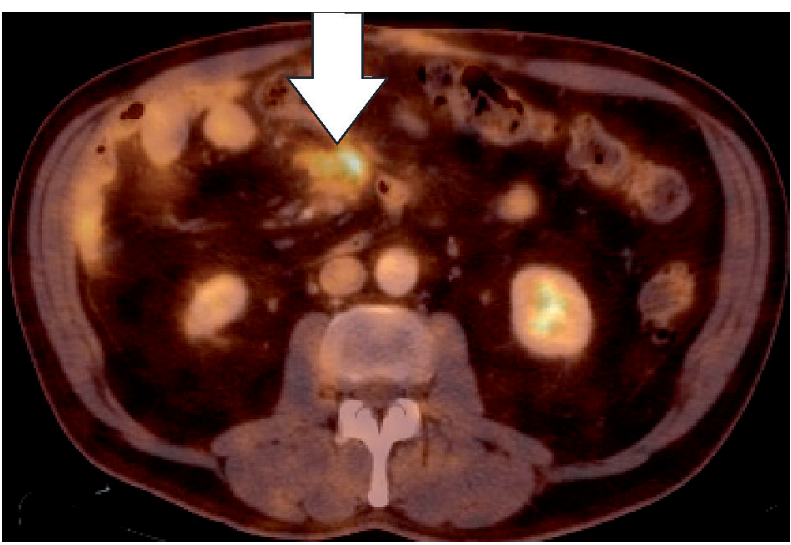

Figure 12. Encapsulated fat necrosis. Axial computed tomography image (Figure 11) and axial fused PET-CT image (Figure 12) show a encapsulated lesion with increased FDG-uptake (white arrow), mimicking metastatic disease in a patient with known colon cancer

es: chronic nonspecific inflammation, fat necrosis, and fibrosis. If inflammation and fat necrosis are predominant, the condition is known as mesenteric panniculitis. When fibrosis and retraction are the principal findings, it is called retractile mesenteritis [15]. The imaging appearance of mesenteric panniculitis depends on the predominant findings (fat necrosis, inflammation, or fibrosis). In the case of mesenteric panniculitis, we usually see a mass-like area with heterogeneously increased fat attenuation and interposed linear bands without displacement of the mesenteric vessels (Figures 14-16). In cases of retractile mesenteritis, it presents as a homogeneous mass of soft tissue density, often with calcifications [15]. [16]:

The classic CT signs (Coulier's criteria) are the following

- the presence of a "mass effect" on neighbouring bowel loops;

- mesenteric fat tissue with high attenuation;

- the presence of lymph nodes;

- a hypoattenuating "halo sign" surrounding the vessels and lymph nodes;

- a hyperattenuating pseudocapsule.

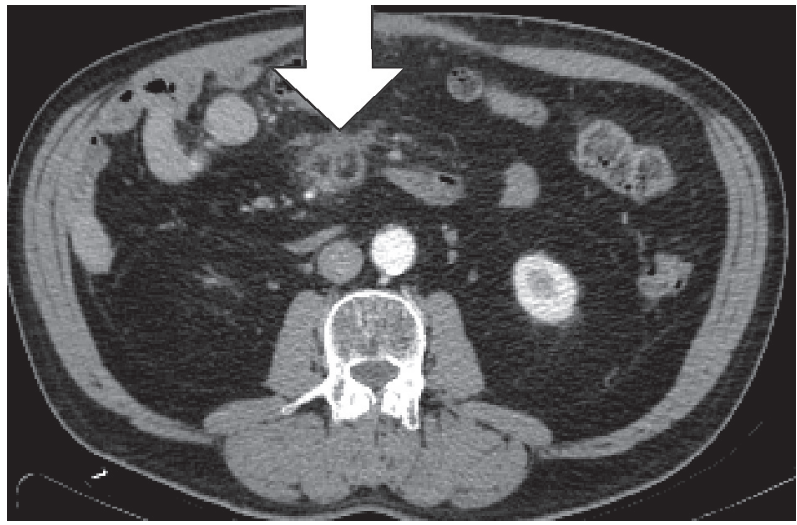

Figure 11. Encapsulated fat necrosis. Axial computed tomography image (Figure 11) and axial fused PET-CT image (Figure 12) show an encapsulated lesion with increased FDG-uptake (white arrow), mimicking metastatic disease in a patient with known colon cancer

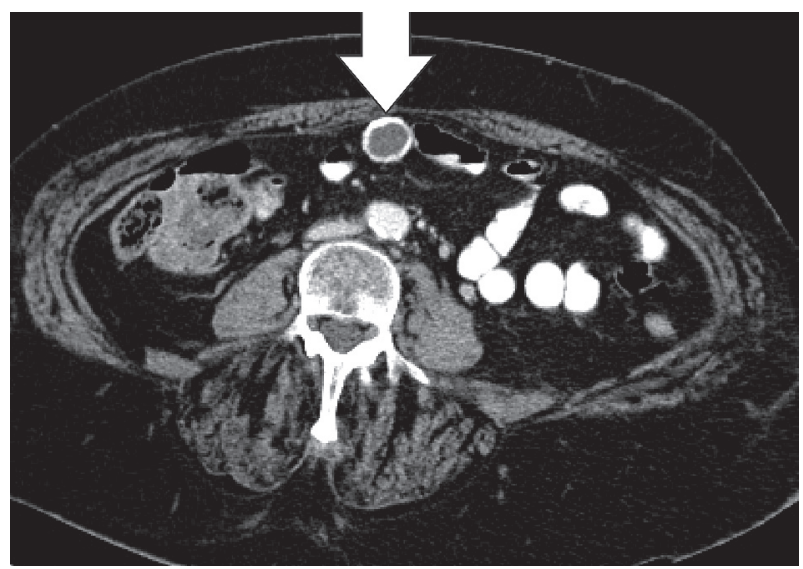

Figure 13. Encapsulated fat necrosis. Axial computed tomography image. Lesions either resolve completely or persist and develop a calcified rim (white arrow), as in this patient

Mesenteric panniculitis is considered when at least three of the five criteria are present.

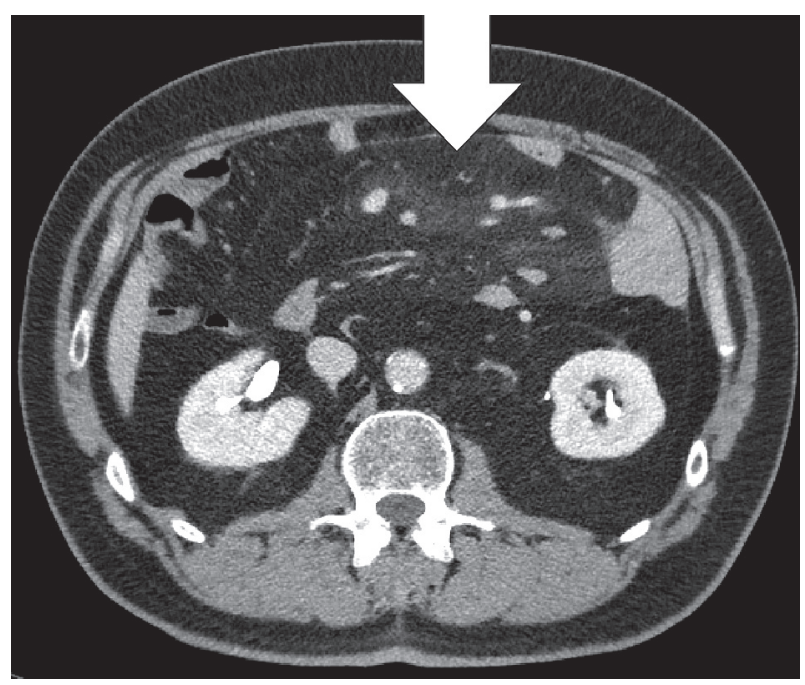

Figure 14. Mesenteric panniculitis. Axial computed tomography image reveals an area of heterogeneously increased fat attenuation that does not displace the surrounding mesenteric vascular structures (white arrow) 


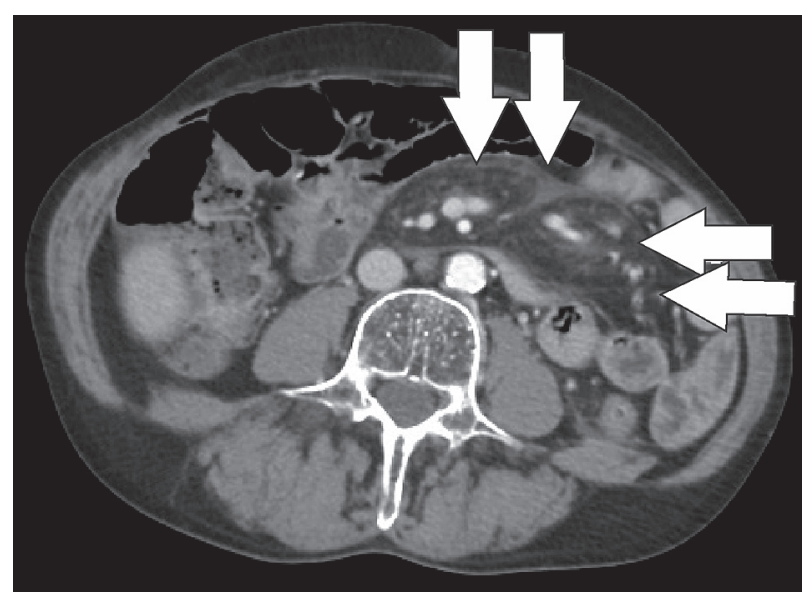

Figure 15. Mesenteric panniculitis. Axial computed tomography image of another patient demonstrates the "tumoral pseudocapsule" sign and a mass effect on adjacent bowel loops (white arrows)

\section{Heterotopic mesenteric ossification}

Heterotopic ossification occurs when of bone develops in the soft tissues. It is a distinct entity from dystrophic calcification, which develops after deposition of calcium salts in the absence of osteoblastic activity [17]. It most commonly occurs in the muscles of the extremities, where it is known as myositis ossificans. Heterotopic mesenteric ossification is a rare complication in patients who are subjected to abdominal trauma or surgery. It can result in complications such as bowel obstruction or fistulas [18]. Classical features include multiple linear and branching, high-attenuation structures within the mesentery, and omentum (Figure 17) [18]. These high-attenuating structures will calcify over time and develop a discernible trabecular and cortical architecture (Figure 18). Differentiation from other entities such as oral contrast leakage, IV contrast extravasation, retained surgical material, or osseous malignancy is vital [17].

\section{Pseudolipoma of the capsule of Glisson}

Pseudolipoma is defined as an encapsulated lesion containing degenerated fat. In the case of a pseudolipoma of the capsule of Glisson, it is a dislodged colonic epiploic appendix that is attached to the liver capsule, often
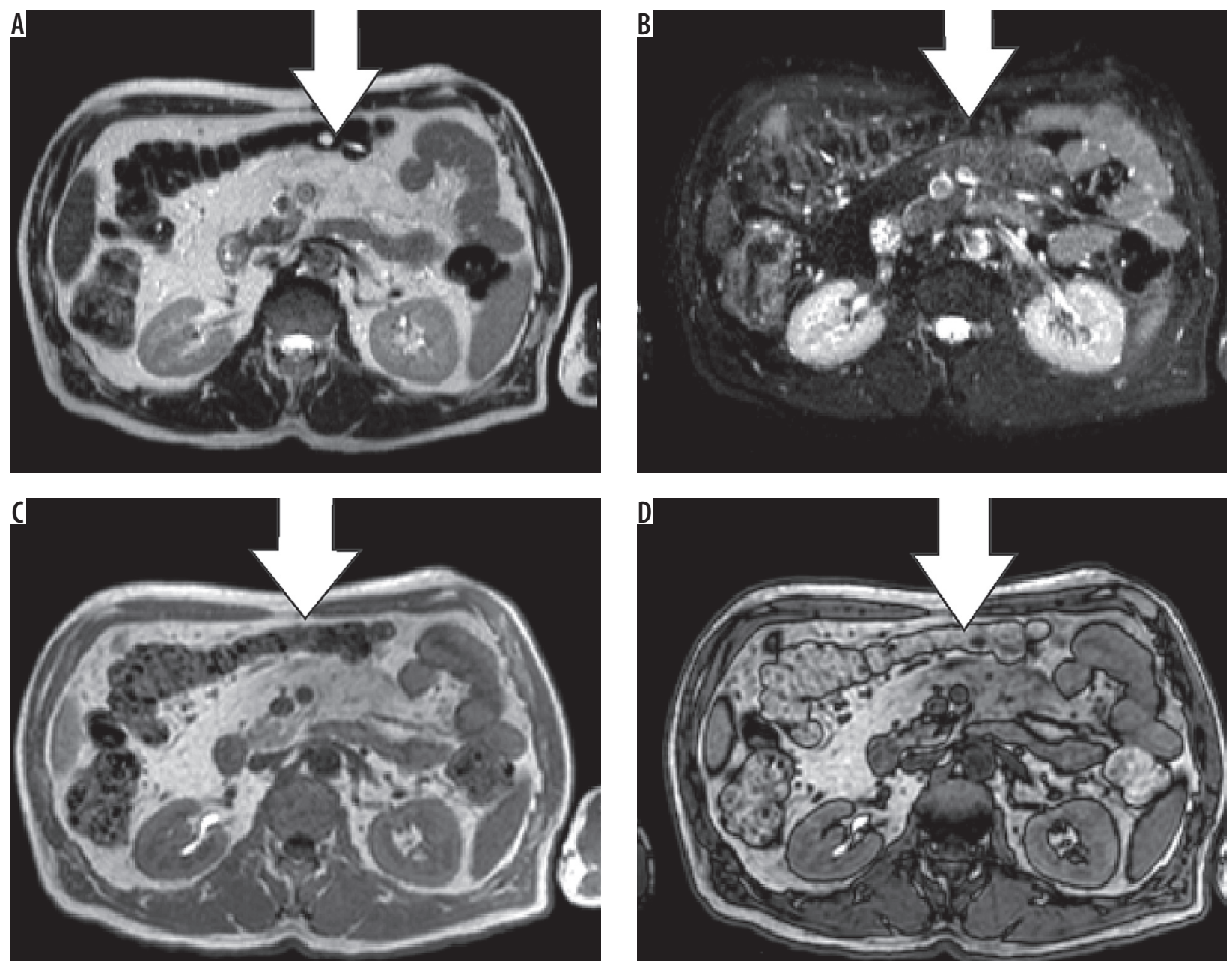

Figure 16. Mesenteric panniculitis. Axial T2 (A), T2 FS (B), in-phase (C), and opposed-phase (D) sequences. While hardly visible on T2, the panniculitis stands out on the other sequences, compared to the normal mesenteric fat (white arrows) 


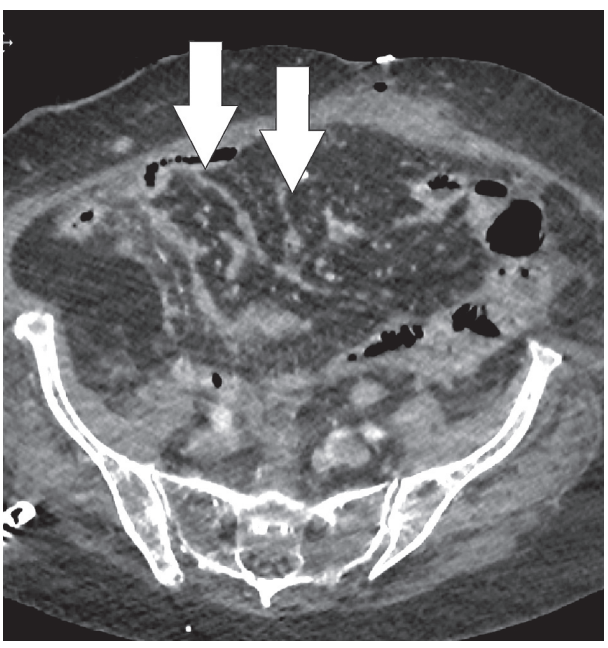

Figure 17. Heterotopic mesenteric ossification. Axial computed tomography image depicts non-specific stranding (white arrows) in the mesentery 1 week after partial colectomy

secondary to prior surgery [19]. On CT, it appears as a well-circumscribed nodule on the liver surface with a centre of fat or soft-tissue attenuation. The size of the reported pseudolipomas ranges from 0.4 to $2.0 \mathrm{~cm}$ (Figures 19,20). The pseudolipoma can be confused with metastatic disease. Another differential diagnosis to consider is a solitary necrotic nodule of the liver [20].

\section{Peritoneal carcinomatosis}

The peritoneum is a common location for metastatic disease. In the case of widespread peritoneal metastases, the term peritoneal carcinomatosis can be used. Peritoneal metastases are mainly seen in gastric and ovarian malignancies. Smooth or nodular thickening of the peritoneum and leaves of the mesentery is the most characteristic presentation,

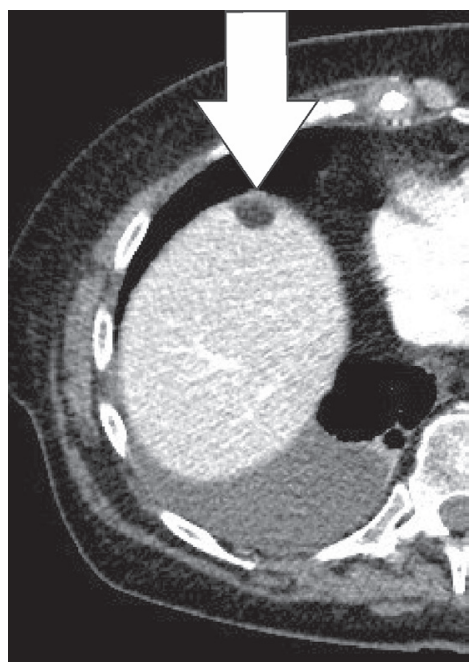

Figure 19. Pseudolipoma of the capsule of Glisson. Axial computed tomography images of a patient, 1 prior to and 1 after sigmoid resection. Appearance of a small, fatty lesion adjacent to the anterior liver capsule (white arrow)

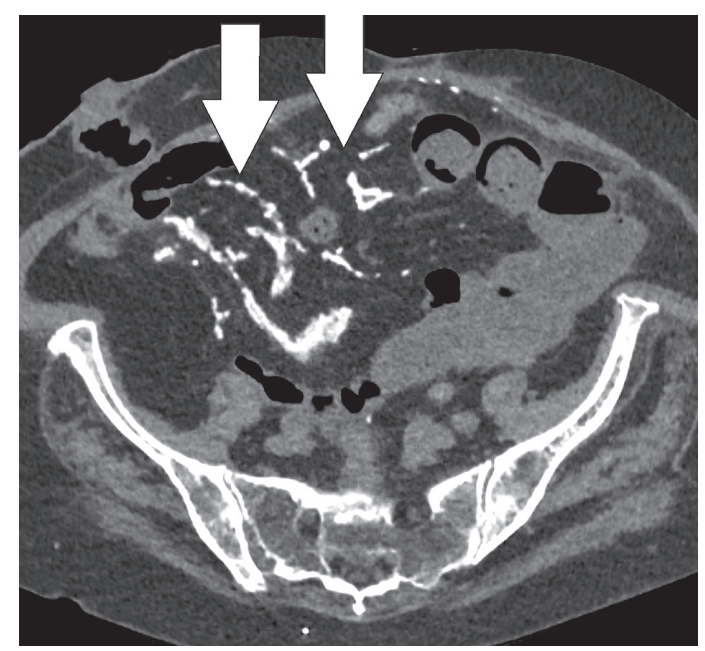

Figure 18. Heterotopic mesenteric ossification. Axial computed tomography image of the same patient 3 months later demonstrate the appearance of calcified linear structures (white arrows) where the non-specific stranding was located

as well as omental nodules and peritoneal enhancement. A stellate appearance of the mesentery may be encountered [21]. A moderate to large volume of ascites is also a frequent finding in peritoneal carcinomatosis. When features atypical of pancreatic fat saponification or mesenteric panniculitis are present or if the clinical history does not coincide with the imaging findings, peritoneal carcinomatosis should be included in the differential diagnosis [22].

\section{Conclusions}

Different pathological processes can lead to intra-abdominal necrosis or inflammation. Lesions originating from the abdominal fat are a common finding in abdominal imaging. Because of the benign and self-limiting nature

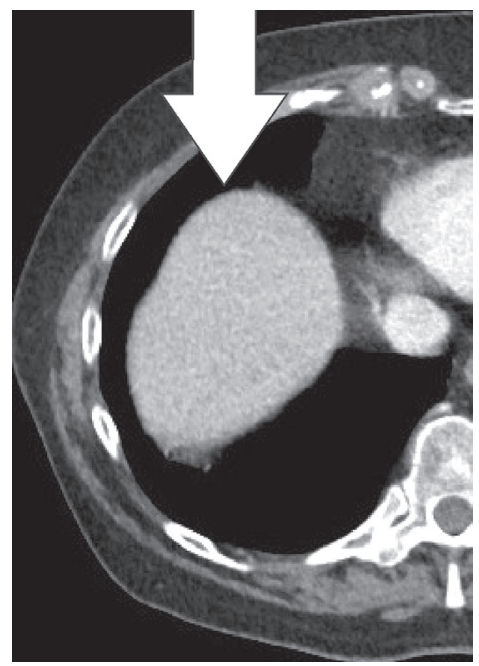

Figure 20. Pseudolipoma of the capsule of Glisson. Axial computed tomography images of a patient, 1 prior to and 1 after sigmoid resection. Appearance of a small, fatty lesion adjacent to the anterior liver capsule (white arrow) 
of these pathologies, surgical intervention is rarely necessary. Most of the lesions present with typical imaging characteristics, and, combined with the clinical history, it is often possible to make the correct diagnosis. It is important to recognise the imaging features and acknowledge them as 'do not touch' lesions. When atypical imaging features are present, such as ascites, peritoneal nodules, or a fast-growing mesenteric mass, malignant disease should be considered.

\section{Conflict of interest}

The authors report no conflict of interest.

\section{References}

1. McClure MJ, Khalili K, Sarrazin J, Hanbidge A. Radiological features of epiploic appendagitis and segmental omental infarction. Clin Radiol 2001; 56: 819-827.

2. Danielson K, Chernin MM, Amberg JR, et al. Epiploic appendicitis: CT characteristics. J Comput Assist Tomogr 1986; 10: 142-143.

3. Rioux M, Langis P. Primary epiploic appendagitis: clinical, US, and CT findings in 14 cases. Radiology 1994; 191: 523-526.

4. Jeon YS, Lee JW, Cho SG. Is it from the mesentery or the omentum? MDCT features of various pathologic conditions in intraperitoneal fat planes. Surg Radiol Anat 2009; 31: 3-11.

5. Grattan-Smith JD, Blews DE, Brand T. Omental infarction in pediatric patients: sonographic and CT findings. AJR Am J Roentgenol 2002; 178: 1537-1539.

6. Kamaya A, Federle MP, Desser TS. Imaging manifestations of abdominal fat necrosis and its mimics. Radiographics 2011; 31: 20212034.

7. Stella DL, Schelleman TG. Segmental infarction of the omentum secondary to torsion: sonography and computed tomography diagnosis. Australas Radiol 2000; 44: 212-215.

8. Balthazar EJ, Lefkowitz RA. Left-sided omental infarction with associated omental abscess: CT diagnosis. J Comput Assist Tomogr 1993; 17: 379-381.

9. Aho HJ, Sternby B, Nevalainen TJ. Fat necrosis in human acute pancreatitis: an immunohistological study. Acta Pathol Microbiol Immunol Scand [A] 1986; 94: 101-105.

10. Franco-Pons N, Gea-Sorlí S, Closa D. Release of inflammatory mediators by adipose tissue during acute pancreatitis. J Pathol 2010; 221: 175-182.

11 Schmidt-Hermes HJ, Loskant G. Calcified fat necrosis of the female breast [in German]. Med Welt 1975; 26: 1179-1180.
12. Brooke BS, Choti MA. Image of the month. Encapsulated fat necrosis. Arch Surg 2011; 146: 1447-1448.

13. Hurt MA, Santa Cruz DJ. Nodular-cystic fat necrosis: a reevaluation of the so-called mobile encapsulated lipoma. J Am Acad Dermatol 1989; 21 (3 Pt 1): 493-498.

14. McLaughlin PD, Filippone A, Maher MM. The "misty mesentery": mesenteric panniculitis and its mimics. AJR Am J Roentgenol 2013; 200: W116-W123.

15. Horton KM, Lawler LP, Fishman EK. CT findings in sclerosing mesenteritis (panniculitis): spectrum of disease. Radiographics 2003; 23: 1561-1567.

16. Daskalogiannaki M, Voloudaki A, Prassopoulos P, et al. CT evaluation of mesenteric panniculitis: prevalence and associated diseases. AJR Am J Roentgenol 2000; 174: 427-431.

17. Ferreira C, Gomes C, Melo A, et al. Heterotopic mesenteric and abdominal wall ossification - two case reports in one institution. Int J Surg Case Rep 2017; 37: 22-25.

18. Davis AK, Kuhls DA, Wulff R, et al. Heterotopic ossification after blunt abdominal trauma. J Trauma 2008; 65: 1536-1539.

19. Prasad SR, Wang $\mathrm{H}$, Rosas $\mathrm{H}$, et al. Fat-containing lesions of the liver: radiologic-pathologic correlation. Radiographics 2005; 25 : 321-331.

20. Anderson SW, Kruskal JB, Kane RA. Benign hepatic tumors and iatrogenic pseudotumors. Radiographics 2009; 29: 211-229.

21. Cabral F, Krajewski K, Kim K, et al. Peritoneal lymphomatosis: CT and PET/CT findings and how to differentiate between carcinomatosis and sarcomatosis. Cancer Imaging 2013; 13: 162-170.

22. Smith JP, Arnoletti JP, Varadarajulu S, Morgan DE. Post-pancreatitis fat necrosis mimicking carcinomatosis. Radiol Case Rep 2015; 3: 192 . 